\title{
Comparison of the transport properties of high quality AIGaN/AIN/GaN and AllnN/AIN/GaN two-dimensional electron gas heterostructures
}

\author{
Remziye Tülek, ${ }^{1}$ Aykut Ilgaz, ${ }^{1}$ Sibel Gökden, ${ }^{1}$ Ali Teke, ${ }^{1, a)}$ Mustafa K. Öztürk, ${ }^{2}$ \\ Mehmet Kasap, ${ }^{2}$ Süleyman Özçelik, ${ }^{2}$ Engin Arslan, ${ }^{3}$ and Ekmel Özbay ${ }^{3}$ \\ ${ }^{1}$ Department of Physics, Faculty of Science and Arts, Balıkesir University, Çağlş Kampüsü, \\ 10145 Balıkesir, Turkey \\ ${ }^{2}$ Department of Physics, Faculty of Science and Arts, Gazi University, Teknikokullar, 06500 Ankara, Turkey \\ ${ }^{3}$ Department of Physics, Department of Electrical and Electronics Engineering, Nanotechnology \\ Research Center-NANOTAM, Bilkent University, 06800 Ankara, Turkey
}

(Received 24 July 2008; accepted 20 August 2008; published online 7 January 2009)

\begin{abstract}
The transport properties of high mobility AlGaN/AlN/GaN and high sheet electron density AlInN/ AlN/GaN two-dimensional electron gas (2DEG) heterostructures were studied. The samples were grown by metal-organic chemical vapor deposition on $c$-plane sapphire substrates. The room temperature electron mobility was measured as $1700 \mathrm{~cm}^{2} / \mathrm{V} \mathrm{s}$ along with $8.44 \times 10^{12} \mathrm{~cm}^{-2}$ electron density, which resulted in a two-dimensional sheet resistance of $435 \Omega / \square$ for the $\mathrm{Al}_{0.2} \mathrm{Ga}_{0.8} \mathrm{~N} / \mathrm{AlN} / \mathrm{GaN}$ heterostructure. The sample designed with an $\mathrm{Al}_{0.88} \mathrm{In}_{0.12} \mathrm{~N}$ barrier exhibited very high sheet electron density of $4.23 \times 10^{13} \mathrm{~cm}^{-2}$ with a corresponding electron mobility of $812 \mathrm{~cm}^{2} / \mathrm{V} \mathrm{s}$ at room temperature. A record two-dimensional sheet resistance of $182 \Omega / \square$ was obtained in the respective sample. In order to understand the observed transport properties, various scattering mechanisms such as acoustic and optical phonons, interface roughness, and alloy disordering were included in the theoretical model that was applied to the temperature dependent mobility data. It was found that the interface roughness scattering in turn reduces the room temperature mobility of the $\mathrm{Al}_{0.88} \mathrm{In}_{0.12} \mathrm{~N} / \mathrm{AlN} / \mathrm{GaN}$ heterostructure. The observed high $2 \mathrm{DEG}$ density was attributed to the larger polarization fields that exist in the sample with an $\mathrm{Al}_{0.88} \operatorname{In}_{0.12} \mathrm{~N}$ barrier layer. From these analyses, it can be argued that the AlInN/AIN/GaN high electron mobility transistors (HEMTs), after further optimization of the growth and design parameters, could show better transistor performance compared to AlGaN/AlN/GaN based HEMTs. (C) 2009 American Institute of Physics. [DOI: 10.1063/1.2996281]
\end{abstract}

\section{INTRODUCTION}

$\mathrm{Al}(\mathrm{In}) \mathrm{GaN} /(\mathrm{In}) \mathrm{GaN}$ based high electron mobility transistors (HEMTs) have recently attracted a great deal of attention for high-frequency and high-power microwave applications because nitride based material systems have fundamental physical properties such as a large band gap, large breakdown field, and strong spontaneous and piezoelectric polarization fields. ${ }^{1}$ To improve the performance of devices, various barrier and channel alternatives have been used in nitride based HEMTs. ${ }^{2-6}$ Among them, the most well studied structure is the $\mathrm{AlGaN} / \mathrm{GaN}$ with a two-dimensional electron gas (2DEG) formed at the heterointerface, which is induced by piezoelectric and spontaneous polarizations. ${ }^{7,8}$ Several achievements have been made in AlGaN/GaN HEMT performance by optimizing the growth and design parameters. For example, the introduction of a thin AlN spacer layer at the $\mathrm{AlGaN} / \mathrm{GaN}$ interface increases the carrier density and effectively reduces the alloy scattering of $2 \mathrm{DEG}$ as well as provides better carrier confinement. ${ }^{7,9,10}$ As a design parameter, high aluminum content is desirable in order to increase the polarization induced charge density and the carrier confinement in the channel. ${ }^{8}$ However, when a higher

\footnotetext{
${ }^{\text {a) }}$ Author to whom correspondence should be addressed. Electronic mail: ateke@balikesir.edu.tr.
}

aluminum content $(>30 \%)$ is used in an AlGaN barrier, the quality of the layer becomes worse in turn resulting in a significant reduction in electron mobility. Consequently, AlGaN/GaN based HEMTs are designed with a trade-off between a high electron mobility $\left(\mu_{e}\right)$, typically $1600 \mathrm{~cm}^{2} / \mathrm{V} \mathrm{s}$, and a high sheet carrier density $\left(n_{s}\right)$, typically $1.5 \times 10^{13} \mathrm{~cm}^{-2}$, providing a two-dimensional sheet resistance $R_{s}$, typically $250 \Omega / \square .{ }^{11}$

In recent years, an alternative approach wherein the Al$\mathrm{GaN}$ layer is replaced by an AlInN barrier has been implemented for improving the HEMT performance after the original proposal of Kuzmík. ${ }^{12}$ The advantage of using an AlInN barrier is to adjust the composition of the alloys to obtain a lattice or polarization matched heterostructure. When the indium composition is set to $\sim 18 \%$ the alloy and $\mathrm{GaN}$ is latticed matched. The polarization charge is, therefore, completely determined by spontaneous polarization since the structure is free of strain and the piezoelectric polarization is zero. The HEMTs with an AlInN barrier layer were essentially predicted to provide higher carrier densities than an AlGaN barrier layer. ${ }^{13}$ If the mobility of the former is kept at the same level with that of the latter, the conductivity performance of the AlInN based devices would be higher, exploring to high power and high frequency transistor operations. However, from the epitaxial point of view, there exists a major difficulty in growing an AlInN based structure be- 
cause the growth of AlN and $\mathrm{InN}$ requires different growth temperatures. Therefore, the formation of AlInN with the variance of the composition in a controlled way is not straightforward. However, reports on AlInN and AlInN/GaN heterostructures in the literature are rapidly increasing. ${ }^{14-18}$ Recently, Gonschorek et al. ${ }^{5}$ reported a mobility value of $1170 \mathrm{~cm}^{2} / \mathrm{V}$ s along with $2.6 \times 10^{13} \mathrm{~cm}^{-2} 2 \mathrm{DEG}$ density for an undoped nearly lattice-matched AlInN/AlN/GaN heterostructure. The corresponding two-dimensional sheet resistance was reported as $210 \Omega / \square$. Furthermore, of these successful results reported in the literature there are only a few reports in terms of the detailed analysis of the transport characteristics of AlInN-based HEMTs., ${ }^{5,19}$

In the present work, we investigated and compared the transport properties of high quality $\mathrm{AlGaN} / \mathrm{AlN} / \mathrm{GaN}$ and AlInN/AlN/GaN heterostructures using temperature dependent Hall effect measurements. Analytical models were applied to the experimental results in order to understand the scattering mechanism that governs the performance of devices in a temperature range of $30-300 \mathrm{~K}$. If the scattering mechanisms that are dominant for high-density 2DEGs can be identified, it will guide the modifications to the growth and/or the layer structure that will be necessary to further improve the conductivity. This work has emphasized that the AlInN/AlN/GaN HEMT structure after the further optimization of the growth and design parameters could show better transistor performance compared to $\mathrm{AlGaN} / \mathrm{GaN}$ based HEMTs.

\section{EXPERIMENTAL DETAILS}

The samples $\quad \mathrm{Al}_{0.2} \mathrm{Ga}_{0.8} \mathrm{~N} / \mathrm{AlN} / \mathrm{GaN}$ and $\mathrm{Al}_{0.88} \mathrm{In}_{0.12} \mathrm{~N} / \mathrm{AlN} / \mathrm{GaN}$ were grown on $c$-plane (0001) sapphire $\left(\mathrm{Al}_{2} \mathrm{O}_{3}\right)$ substrates in a low-pressure metal-organic chemical vapor deposition reactor (Aixtron 200/4 HT-S). Trimethylgallium (TMGa), trimethylaluminum (TMAl), trimethylindium (TMIn), and ammonia $\left(\mathrm{NH}_{3}\right)$ were used as $\mathrm{Ga}$, $\mathrm{Al}$, In, and $\mathrm{N}$ precursors, respectively. ${ }^{20}$ Prior to the epitaxial growth, substrate was annealed at $1100{ }^{\circ} \mathrm{C}$ for $10 \mathrm{~min}$ in a nitrogen environment to remove the surface oxides. For both samples, the growth of HEMT structures was initiated with a 15-nm-thick low temperature AlN nucleation layer at a temperature of $650{ }^{\circ} \mathrm{C}$. Then, a $0.5 \mu \mathrm{m}$ thick high temperature (HT) AlN buffer layer was grown at $1150{ }^{\circ} \mathrm{C}$. A $2 \mu \mathrm{m}$ thick nominally undoped $\mathrm{GaN}$ layer was then grown at $1050{ }^{\circ} \mathrm{C}$. In order to reduce the alloy disorder scattering, a $1.2-1.3 \mathrm{~nm}$ thick HT AIN spacer layer was grown at a temperature of $1150{ }^{\circ} \mathrm{C}$. AlGaN and AlInN barrier layers were deposited on AlN spacer layer at growth temperatures of 1050 and $800{ }^{\circ} \mathrm{C}$, respectively. The growths were finished by growing a $3 \mathrm{~nm}$ thick GaN cap layer at a temperature of $1050{ }^{\circ} \mathrm{C}$.

The crystalline quality and dislocation densities of the $\mathrm{GaN}$ layers and the $\mathrm{Al}$ and In compositions of the barrier layers were determined by high-resolution $\mathrm{x}$-ray diffraction (XRD). The XRD was performed by using a Bruker D-8 high-resolution diffractometer system, delivering $\mathrm{Cu} K \alpha 1$ $\left(\begin{array}{ll}1.540 & \AA\end{array}\right)$ radiation using a prodded mirror and four-bounce $\mathrm{Ge}(220)$ symmetric monochromator.

Variable temperature Hall measurements were used to

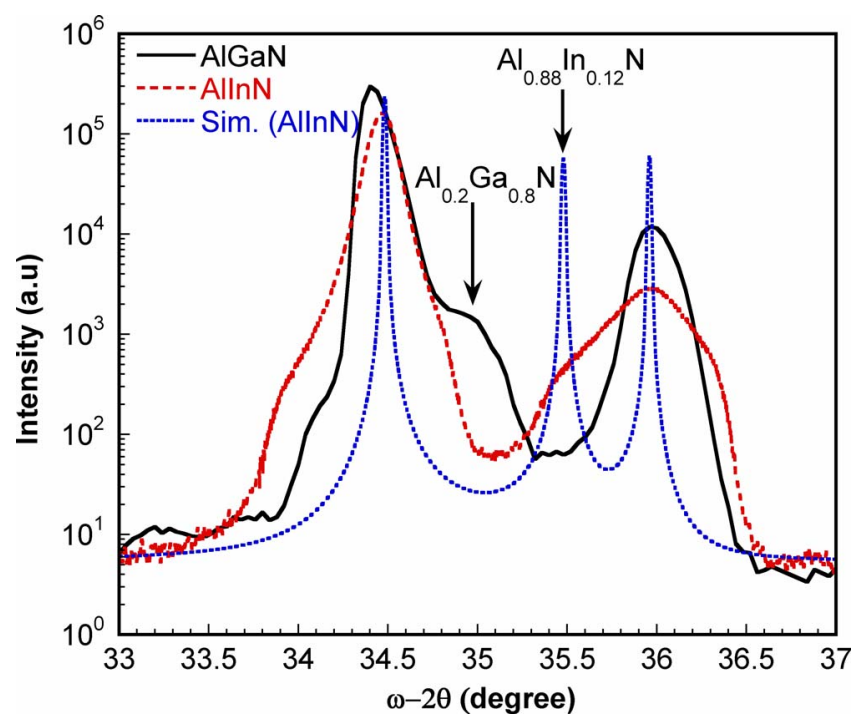

FIG. 1. (Color online) High resolution XRD (0002) $\omega-2 \theta$ scans of AlGaN/ AlN/GaN and AlInN/AlN/GaN heterostructures along with the simulation curve of the latter obtained for the In composition of 0.12 in AlInN barrier layer.

measure the 2DEG mobility and the sheet carrier density for both samples. For the Hall effect measurements, square shaped samples in van der Pauw geometry were prepared with four evaporated $\mathrm{Ti} / \mathrm{Al} / \mathrm{Ni} / \mathrm{Au}$ triangular Ohmic contacts in the corners. Using gold wires and In soldering, the electrical contacts were made and their Ohmic behavior was confirmed by the current-voltage $(I-V)$ characteristics. The measurements were performed at various temperatures over a temperature range of $30-300 \mathrm{~K}$ by using a Lake Shore Hall effect measurement system.

\section{EXPERIMENTAL RESULTS}

XRDs were performed to determine the alloy compositions and dislocation densities for both $\mathrm{AlGaN} / \mathrm{AlN} / \mathrm{GaN}$ and AlInN/AlN/GaN HEMTs. The XRD data were collected on the (0002) and (1231) reflections with $\omega-2 \theta$ scans. Figure 1 shows the $\omega-2 \theta$ scan XRD patterns around the (0002) reflection of both samples. The spectra are dominated by the GaN peak at an angle of about $34.5^{\circ}$ originating from the underlying $\mathrm{GaN}$ template. Additional peaks seen at $\sim 36^{\circ}$ were attributed to the AlN buffer and interlayer. The well-resolved peaks (or shoulders) related to $\mathrm{AlGaN}$ and AlInN barrier layers in the AlGaN/AlN/GaN and AlInN/AlN/GaN HEMTs were observed at $\sim 35^{\circ}$ and $35.5^{\circ}$, respectively. In addition, no phase separations are observed in any of the curves, which indicate that both $\mathrm{AlGaN}$ and AlInN layers were grown coherently on the AlN/GaN structures. From the relative XRD peak positions and using lattice constants of $\mathrm{GaN}$, $\mathrm{AlN}$, and InN as given in Table I and Vegard's law, the Al and In compositions were determined to be $20 \%$ and $12 \%$, for AlGaN/AlN/GaN and AlInN/AlN/GaN HEMTs, respectively. The In composition in AlInN barrier layer was also confirmed by using the simulation curve that is superimposed on the measured XRD curve (Fig. 1). From the XRD measurements we also estimated the edge and screw type of the dislocation densities, which are used in the mobility cal- 
TABLE I. The constants used for the calculation of the polarization and sheet carrier density in AlGaN/AlN/GaN and AlInN/AlN/GaN heterostructures.

\begin{tabular}{lccc}
\hline \hline & AlN & GaN & InN \\
\hline$P_{S P}\left(\mathrm{C} / \mathrm{m}^{2}\right)$ & -0.081 & -0.029 & -0.032 \\
$e_{33}\left(\mathrm{C} / \mathrm{m}^{2}\right)$ & 1.46 & 0.73 & 0.97 \\
$e_{31}\left(\mathrm{C} / \mathrm{m}^{2}\right)$ & -0.60 & -0.49 & -0.57 \\
$C_{13}(\mathrm{GPa})$ & 108 & 103 & 92 \\
$C_{33}(\mathrm{GPa})$ & 373 & 405 & 224 \\
$a_{0}(\AA)$ & 3.112 & 3.189 & 3.540 \\
\hline \hline
\end{tabular}

culation of the dislocation scattering. The edge and screw type of the dislocation densities were calculated as 9.5 $\times 10^{8} \mathrm{~cm}^{-2}\left(5.1 \times 10^{8} \mathrm{~cm}^{-2}\right)$ and $5.2 \times 10^{7} \mathrm{~cm}^{-2} \quad(5.4$ $\left.\times 10^{7} \mathrm{~cm}^{-2}\right)$ in the GaN templates for the samples having $\mathrm{AlGaN}(\mathrm{AlInN})$ barrier layers, respectively. One can refer to Ref. 21 for the details of the calculation method of the dislocation density using XRD.

The Hall measurements were performed by loading the samples into a closed-cycle He cryostat, in which the temperature varied between 30 and $300 \mathrm{~K}$. Figure 2 shows the 2DEG sheet density and sheet resistance for both samples. As seen in the figure, the 2DEG sheet densities of 8.44 $\times 10^{12}$ and $4.23 \times 10^{13} \mathrm{~cm}^{-2}$ were obtained for AlGaN/AlN/ $\mathrm{GaN}$ and AlInN/AlN/GaN structures, respectively, at room temperature. They both slightly decrease as the temperature reduces and reaches the values of $7.59 \times 10^{12}$ and 3.55 $\times 10^{13} \mathrm{~cm}^{-2}$ in the above order, respectively, at the lowest temperatures. These temperature behaviors of $2 \mathrm{DEG}$ sheet densities imply that the conduction is dominated nearly exclusively by the carriers at the AlN/GaN heterointerfaces for both HEMT structures. In the same figure it is seen that the corresponding room temperature two-dimensional sheet resistances were measured as 434 and $182 \Omega / \square$ for AlGaN/ AlN/GaN and AlInN/AlN/GaN structures, respectively. According to the best of our knowledge, the sheet resistance of $182 \Omega / \square$ measured for the AlInN/AlN/GaN type of struc-

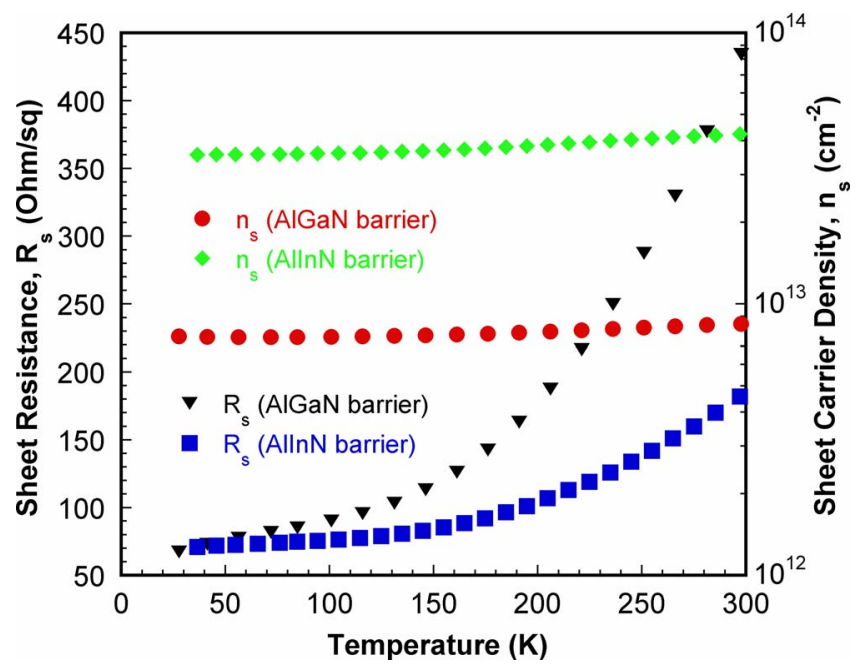

FIG. 2. (Color online) The temperature dependence of measured sheet carrier density and sheet resistance for both $\mathrm{Al}_{0.2} \mathrm{Ga}_{0.8} \mathrm{~N} / \mathrm{AlN} / \mathrm{GaN}$ and $\mathrm{Al}_{0.88} \mathrm{In}_{0.12} \mathrm{~N} / \mathrm{AlN} / \mathrm{GaN}$ heterostructures.

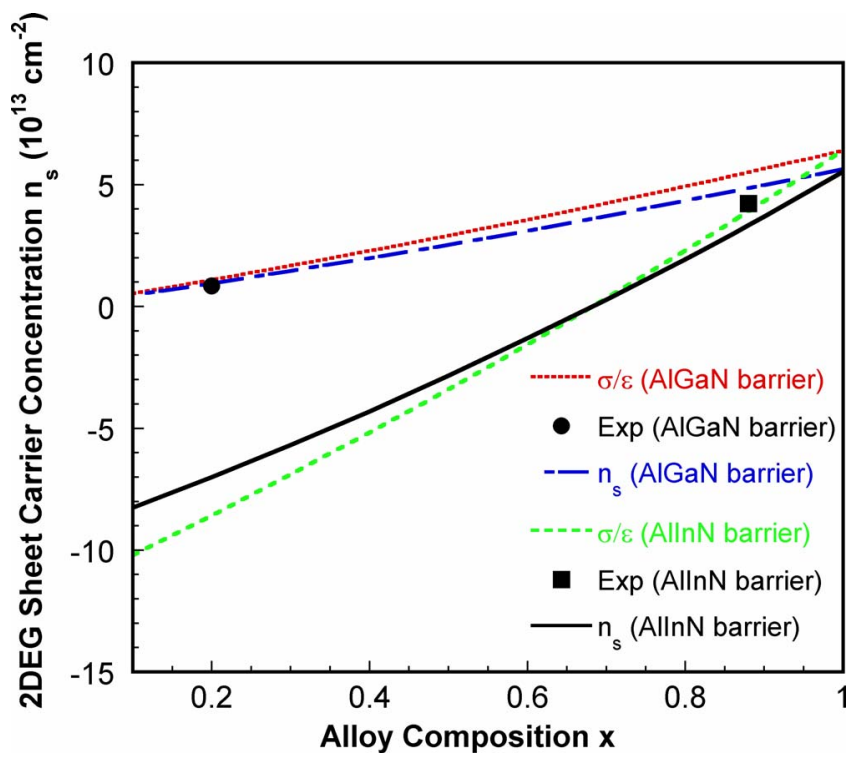

FIG. 3. (Color online) Composition dependence of the maximum sheet carrier concentration of the 2DEG confined at $\mathrm{Al}_{x} \mathrm{Ga}_{1-x} \mathrm{~N} / \mathrm{AlN} / \mathrm{GaN}$ and $\mathrm{Al}_{x} \mathrm{In}_{1-x} \mathrm{~N} / \mathrm{AlN} / \mathrm{GaN}$ interfaces including GaN cap layer and $\mathrm{AlN}$ spacer layer. The total polarization induced bound sheet charges $\sigma / e$ are also plated to see the effect of the $\mathrm{GaN}$ cap layer. For comparison, the experimental sheet carrier densities obtained by Hall measurement at room temperature are also indicated as circle and square for $\mathrm{Al}_{0.2} \mathrm{Ga}_{0.8} \mathrm{~N} / \mathrm{AlN} / \mathrm{GaN}$ and $\mathrm{Al}_{0.88} \mathrm{In}_{0.12} \mathrm{~N} / \mathrm{AlN} / \mathrm{GaN}$, respectively.

ture is the lowest value reported in the literature. This achievement is due to the improvement in the quality of the epilayers and the high sheet carrier density at the heterointerface. As the temperature decreases, they both gradually decrease. The decay of the sheet resistance for the AlInN/AIN/ $\mathrm{GaN}$ structure is faster than that for the AlGaN/AlN/GaN structure due to their temperature dependent Hall mobility characteristics, in which they both cease nearly at the same value of $\sim 70 \Omega / \square$ at low temperatures.

To calculate the $2 \mathrm{DEG}$ sheet concentrations from the polarization induced sheet charge densities and to compare them with the observed sheet charge densities in heterostructures with $\mathrm{AlGaN}$ and AlInN barrier layers, the theory presented by Ambacher et al. ${ }^{8}$ and Asbeck et al. ${ }^{22}$ has been pursued. The constants used in our calculation were taken from Bernardini et al. and Wright ${ }^{23}$ and are shown in Table I. Figure 3 shows the calculated maximum sheet electron densities $n_{s}(x)$ of the 2DEG along with the experimental results located at the $\mathrm{AlN} / \mathrm{GaN}$ interface of the $\mathrm{AlGaN} / \mathrm{AlN} / \mathrm{GaN}$ and AlInN/AlN/GaN HEMT structures. In this calculation, the effect of the AlN spacer layer and GaN cap layer was taken into account. As seen in the figure, although the sign of the sheet carrier is always positive for the whole composition range of the $\mathrm{Al}_{x} \mathrm{Ga}_{1-x} \mathrm{~N}$ barrier, it becomes negative below the Al composition of $\sim 0.7$ for $\mathrm{Al}_{x} \mathrm{In}_{1-x} \mathrm{~N}$ barrier, which implies a possible design of $p$-type HEMT structure that uses an AlInN barrier. For the constant spacer layer, the barrier width and cap layer of $1.2,20$, and $3 \mathrm{~nm}$, in order, and the two-dimensional sheet carrier densities were determined to be $0.95 \times 10^{13}$ and $3.34 \times 10^{13} \mathrm{~cm}^{-2}$ for the $\mathrm{Al}$ compositions of $x=0.2$ and $x=0.88$ (corresponding to $12 \%$ of the In content) for AlGaN/AlN/GaN and AlInN/AlN/GaN HEMTs, respectively. The corresponding sheet carrier concentrations 


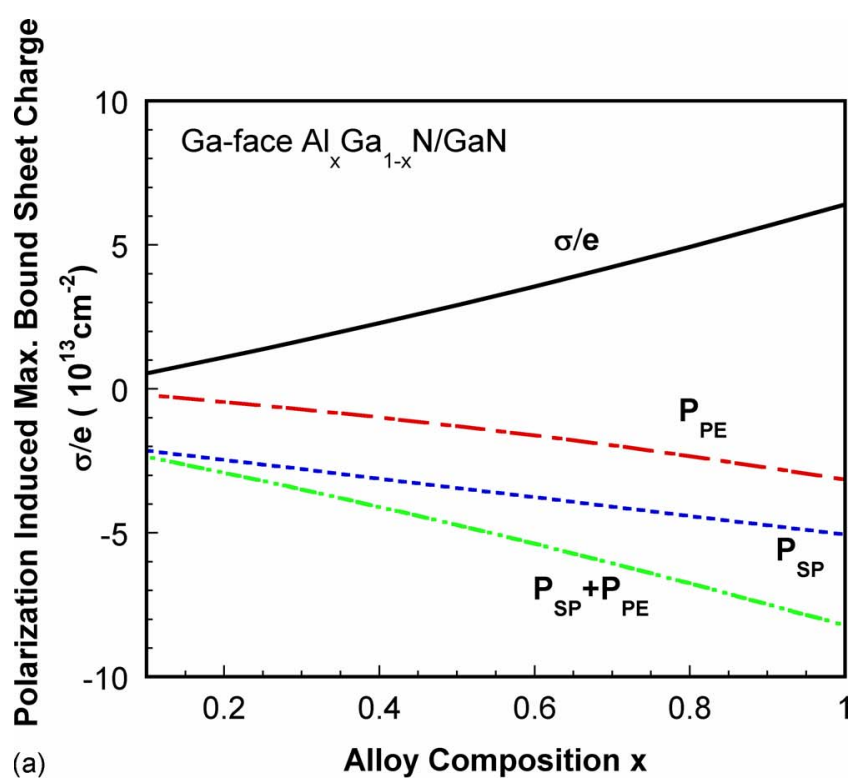

(a)

Alloy Composition $\mathbf{x}$

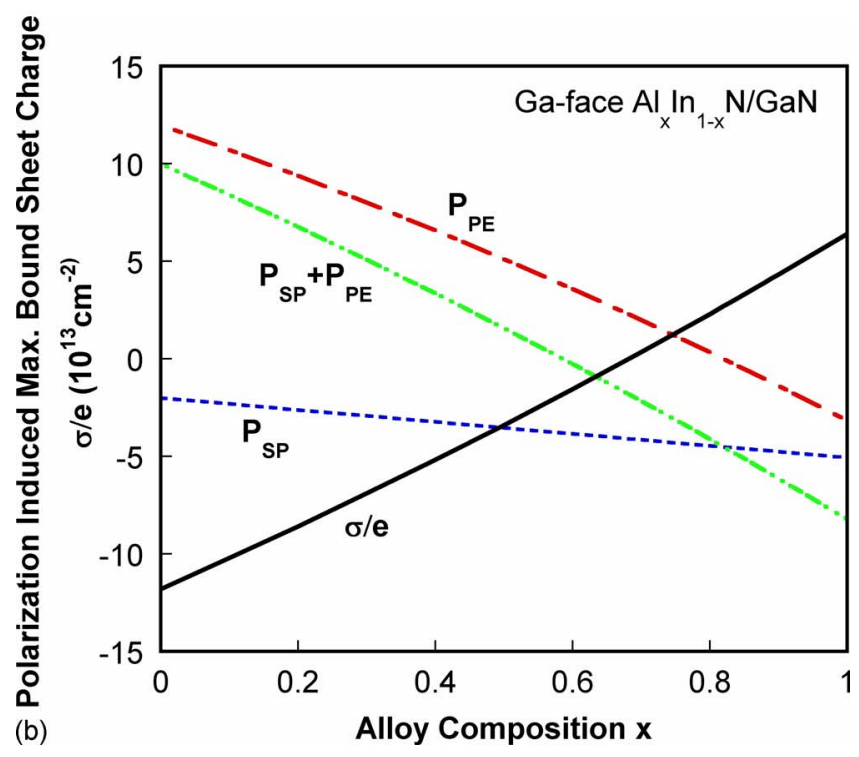

FIG. 4. (Color online) Composition dependence of spontaneous and piezoelectric polarization component of the calculated sheet charge densities at the interfaces of (a) $\mathrm{Al}_{x} \mathrm{Ga}_{1-x} \mathrm{~N} / \mathrm{AlN} / \mathrm{GaN}$ and (b) $\mathrm{Al}_{x} \mathrm{In}_{1-x} \mathrm{~N} / \mathrm{AlN} / \mathrm{GaN}$ heterostructures.

that were experimentally determined by Hall effect measurements were obtained as $0.84 \times 10^{13}$ and $4.23 \times 10^{13} \mathrm{~cm}^{-2}$, which are slightly different from the above values, probably due to the uncertainties in the growth parameters such as layer thicknesses and the exact value of alloy compositions in the real samples or the effect of the nonlinear characteristics of Vegard's law. In Fig. 3, we also plot the polarization induced maximum bound sheet charge $\sigma / e$ versus the alloy composition to identify the effect of the GaN cap layer. The contribution of the GaN cap layer to the overall sheet carrier density is insignificant for the $\mathrm{Al}_{0.2} \mathrm{Ga}_{0.8} \mathrm{~N} / \mathrm{AlN} / \mathrm{GaN}$ heterostructure, while it has a comparably measurable effect for the $\mathrm{Al}_{0.88} \mathrm{Ina}_{0.12} \mathrm{~N} / \mathrm{AlN} / \mathrm{GaN}$ heterostructure.

In order to compare the spontaneous and piezoelectric components of the maximum sheet carrier densities for both samples, we plot the polarization induced bound sheet charge densities separately in Figs. 4(a) and 4(b). For a fixed Al

TABLE II. Values of GaN material constants used in the calculation of scattering mechanisms.

Electron effective mass $\left(m_{0}\right)$

High frequency dielectric constant $\left(\varepsilon_{0}\right)$

Static dielectric constant $\left(\varepsilon_{0}\right)$

LO-phonon energy (meV)

Longitudinal acoustic phonon velocity $(\mathrm{m} / \mathrm{s})$

Density of the crystal $\left(\mathrm{kg} / \mathrm{m}^{3}\right)$

Deformation potential $(\mathrm{eV})$

Piezoelectric constants $\left(\mathrm{C} / \mathrm{m}^{3}\right)$

Elastic constants $\left(\mathrm{N} / \mathrm{m}^{2}\right)$

The electromechanical coupling coefficient

Electron wave vector $\left(\mathrm{m}^{-1}\right)$

Effective Bohr radius in the material $(\AA)$

Lattice constant in the (0001) direction $(\AA)$

The 2D Thomas Fermi wave vector $\left(\mathrm{m}^{-1}\right)$

$$
\begin{gathered}
m^{*}=0.22 \\
\varepsilon_{\infty}=5.35 \\
\varepsilon_{s}=8.9 \\
\hbar \omega=92 \\
v_{L}=6.56 \times 10^{3} \\
\rho=6.15 \times 10^{3} \\
E_{D}=8.3 \\
e_{15}=-0.49 \\
e_{31}=-0.33 \\
e_{33}=0.7 \\
c_{L A}=2.65 \times 10^{11} \\
c_{T A}=0.442 \times 10^{11} \\
K^{2}=0.039 \\
k=7.27 \times 10^{8} \\
a_{B}=23.1 \\
c_{0}=5.185 \\
q_{T F}=8.68 \times 10^{8}
\end{gathered}
$$

composition of 0.88 , the ratio $P_{S P} / P_{P E}$ will be more than two times higher in HEMT with the $\mathrm{Al}_{0.88} \mathrm{In}_{0.12} \mathrm{~N}$ barrier than with the $\mathrm{Al}_{0.88} \mathrm{Ga}_{0.12} \mathrm{~N}$ barrier. Therefore, the necessities of a high $\mathrm{Al}$ content in nitride based HEMT devices for high power and high frequency applications would be accomplished by implementing a slightly off-lattice matched (tensile strain) AlInN barrier layer. As we have shown, a very high sheet carrier concentration $\left(\sim 4 \times 10^{13} \mathrm{~cm}^{-2}\right)$ mainly induced by spontaneous polarization was realized by using the AlInN barrier layer with the $\mathrm{Al}$ composition of 0.88 . In the literature, the reported sheet carrier densities of lattice matched AlInN HEMTs are in the range of (1.2-3.2) $\times 10^{13} \mathrm{~cm}^{-2}$ with various Hall mobilities of $1000-1700 \mathrm{~cm}^{2} / \mathrm{V} \mathrm{s}$, providing the best sheet resistance of about $200 \Omega / \square$. Therefore, we suggest to investigate the AlInN based HEMTs further with a slight tensile strain in order to achieve better conductivity compared to a lattice matched AlInN barrier layer.

Certainly, a higher sheet carrier density is not the only transport parameter in order to accomplish the task that is related to higher conductivity. It is here that we studied the temperature dependent Hall mobilities for both HEMTs with $\mathrm{AlGaN}$ and AlInN barrier layers along with the results of the theoretical model. The model accounts for the major scattering mechanisms such as optical phonon, acoustic phonon through both deformation potential and piezoelectric, interface roughness, background impurity, dislocation, and alloy disordering. The details of the calculations are given in Ref. 24 and references therein. The parameters used in these calculations are taken from Ref. 1 and tabulated in Table II. Since the difference in 2DEG sheet densities at the lowest and highest temperatures is only $10 \%-15 \%$, they are assumed to be constant throughout the whole temperature range for the calculation of the scattering mechanisms. The results are shown in Fig. 5. For the AlGaN/AIN/GaN HEMT structure [Fig. 5(a)], the measured 2DEG Hall mobility is obtained as $1700 \mathrm{~cm}^{2} / \mathrm{V} \mathrm{s}$ at room temperature and reaches $12200 \mathrm{~cm}^{2} / \mathrm{V} \mathrm{s}$ at low temperatures $(30 \mathrm{~K})$. The calculated 

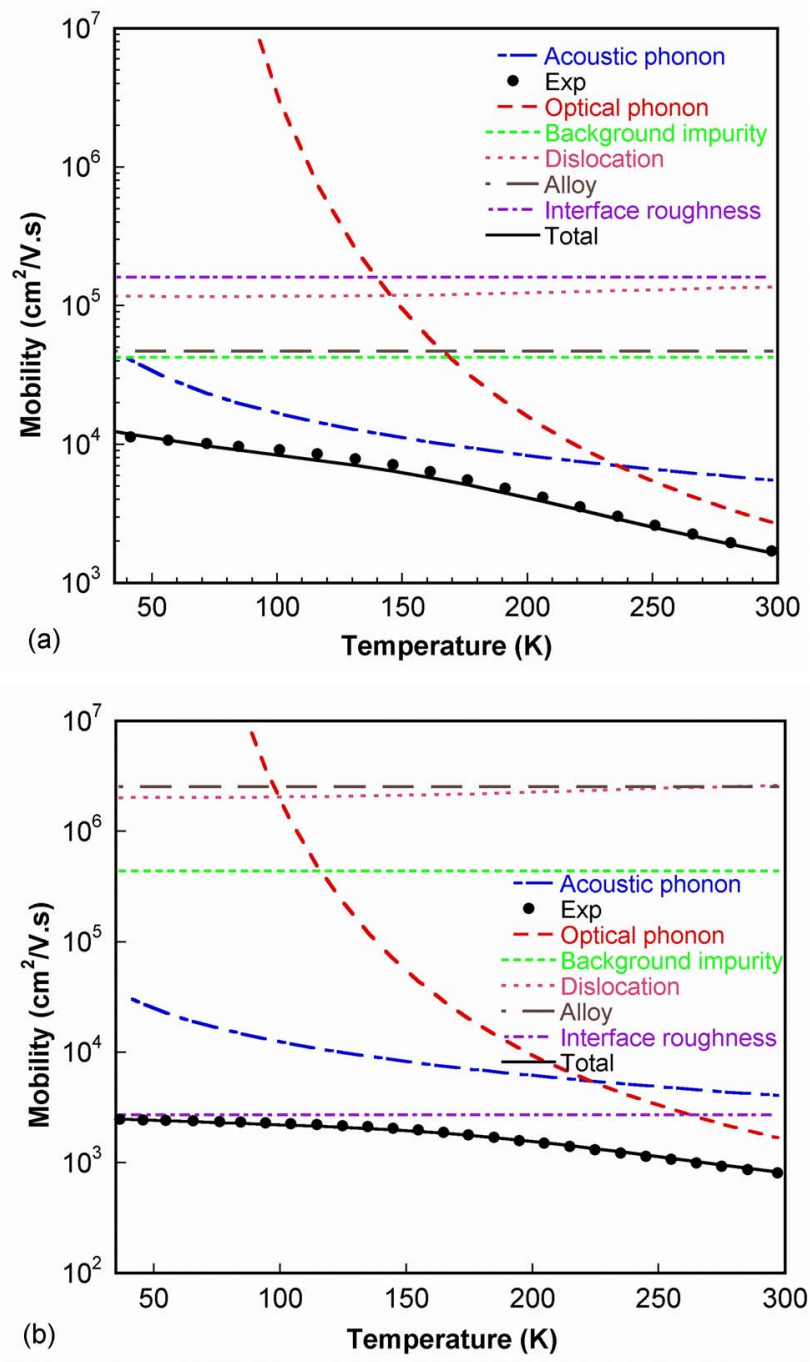

FIG. 5. (Color online) The temperature evolution of the measured Hall mobility in comparison with the theoretical calculations including major scattering mechanisms for (a) $\mathrm{Al}_{0.2} \mathrm{Ga}_{0.8} \mathrm{~N} / \mathrm{AlN} / \mathrm{GaN}$ and (b) $\mathrm{Al}_{0.88} \mathrm{In}_{0.12} \mathrm{~N} / \mathrm{AlN} / \mathrm{GaN}$ heterostructures.

total mobility as a function of the lattice temperature is in very good agreement with the experimental result. As can be clearly seen, HT $(T>200 \mathrm{~K})$ mobility is determined by both acoustic and polar optical phonon scatterings with the increasing strength of the optical phonon component as the temperature increases to room temperature. At moderate temperature ranges, the acoustic phonon scattering through both deformation potential and piezoelectric interactions with nearly equal strength dominates the Hall mobility in the AlGaN/AlN/GaN heterostructure. As the temperature decreases further, the mobility is characterized by the combination of three scattering mechanisms, namely, background impurity, alloy disorder, and acoustic phonon (both components are still nearly equally effective). The experimental and calculated results for 2DEG transport properties indicated that an $\mathrm{AlN}$ spacer layer between the $\mathrm{AlGaN}$ and $\mathrm{GaN}$ layers effectively suppresses alloy disorder scattering. Other mechanisms such as interface roughness scattering and scattering through charged dislocation lines are found to be insignificant for the entire temperature range. In interface roughness scattering, the correlation length and lateral size of the roughness at the AlN/GaN interface were taken to be 230 and $4 \AA$, respectively. The weaknesses of these two scattering mechanisms assure the realization of a high quality $\mathrm{GaN}$ channel with a low dislocation density and a smooth interface.

For the AlInN/AlN/GaN heterostructure [Fig. 5(b)], room temperature Hall mobility is measured as $812 \mathrm{~cm}^{2} / \mathrm{V}$ s. It increases by decreasing temperature and reaches the maximum value of about $2500 \mathrm{~cm}^{2} / \mathrm{V} \mathrm{s}$ at $\sim 40 \mathrm{~K}$. We have again obtained a very good consistency between the temperature dependence of the calculated total mobility data and the experimental results. However, the temperature dependent behavior of the heterostructure with the AlInN barrier layer is profoundly different from that of the sample with the AlGaN barrier layer. As seen in Fig. 5(b) the mobility is nearly determined by the interface roughness scattering at low and moderate temperatures. The correlation length $\Lambda$ and lateral size of roughness $\Delta$ at the AlN/GaN interface were taken to be 170 and $5 \AA$, respectively. In general terms, the mobility increases if $\Delta$ decreases and $\Lambda$ increases. When the fitting parameters are compared between AlGaN/AlN/GaN and AlInN/AlN/GaN heterostructures, it can be argued that the interface of the latter is slightly worse than that of the former. However, this could not explain the observed large difference in the mobility values, especially at low temperatures. The characteristic of the wave function determines the strength of the scattering, which means that the electron scattering is most prominent for the electrons closest to the interface. As the 2DEG density increases, the centroid of the electron distribution shifts to the interface, resulting in a more severe interface roughness scattering. It is totally in agreement with the study of Lisesivdin et al. ${ }^{10}$ who reported the effect of the increase in sheet carrier density due to the increase in barrier thickness as shift in the centroid of the electron distribution toward the interface.

Above $200 \mathrm{~K}$, besides the interface roughness, the polar optical phonon scattering mechanism comes to play along with a small contribution of acoustic phonon scattering. In acoustic phonon scattering, the piezoelectric component is about two times less effective than the deformation potential scattering due to the reduced piezoelectric field in $\mathrm{Al}_{0.88} \mathrm{In}_{0.12} \mathrm{~N}$ and GaN. Since the electron-phonon scattering time constant depends on the electron density in the channel via the wave function as well as the electron distribution statistics, the electron mobility limited by purely acoustic and optical phonon scattering (2700 and $1700 \mathrm{~cm}^{2} / \mathrm{V} \mathrm{s}$, respectively) in the AlInN/AlN/GaN heterostructure is much less than that (5500 and $2700 \mathrm{~cm}^{2} / \mathrm{V} \mathrm{s}$, respectively) in the $\mathrm{AlGaN} / \mathrm{AlN} / \mathrm{GaN}$ heterostructure. Additionally, the background impurity, alloy disorder, and dislocation scatterings are even much less effective because of the efficient screenings arising from the high 2DEG density. The higher band edge discontinuity between $\mathrm{Al}_{0.88} \mathrm{In}_{0.12} \mathrm{~N}$ and GaN compared to the $\mathrm{Al}_{0.2} \mathrm{Ga}_{0.8} \mathrm{~N}$ and $\mathrm{GaN}$ systems is an additional factor in determining the effectiveness of background impurity scattering.

If we assume that the interface roughness scattering is completely eliminated by growing a better AlInN/AlN/GaN interface, we expect much higher electron mobility that is 
mainly limited by an intrinsic optical phonon with a secondarily effective acoustic phonon scattering. In this particular scenario, we would expect to have the electron mobility of about $1200 \mathrm{~cm}^{2} / \mathrm{V} \mathrm{s}$ at room temperature. If the current 2DEG density is assumed to be satisfied, we would have a two-dimensional sheet resistance of only about $120 \Omega / \square$. This value can be reduced further by playing with the In composition in an AlInN layer providing a slightly lower carrier density, but much higher mobility with the enhanced AlN/GaN interface.

\section{CONCLUSIONS}

We studied the transport properties of $\mathrm{Al}_{0.2} \mathrm{Ga}_{0.8} \mathrm{~N} / \mathrm{AlN} / \mathrm{GaN}$ and $\mathrm{Al}_{0.88} \mathrm{In}_{0.12} \mathrm{~N} / \mathrm{AlN} / \mathrm{GaN}$ heterostructures comparatively by using temperature dependent Hall measurements. A very high $2 \mathrm{DEG}$ density of 4.23 $\times 10^{13} \mathrm{~cm}^{-2}$ with a record two-dimensional sheet resistance of $182 \Omega / \square$ was obtained for a heterostructure with an $\mathrm{Al}_{0.88} \mathrm{In}_{0.12} \mathrm{~N}$ barrier layer. The scattering mechanisms were successfully analyzed and the dominant scattering mechanisms in the low and HT regimes were determined for both heterostructures. The major conclusion from the detailed analysis of the theoretical model is that if the growth conditions and design parameters can be modified further in order to reduce the roughness of the $\mathrm{Al}_{x} \mathrm{In}_{1-x} \mathrm{~N} / \mathrm{AlN} / \mathrm{GaN}$ HEMT structure, even higher electron mobilities that result in lower two-dimensional sheet resistances are possible.

\section{ACKNOWLEDGMENTS}

This work is supported by the State of Planning Organization of Turkey under Grant No. 2001K120590, by TUBITAK under Project Nos. 104E090, 105E066, 105A005, 106E198, and 106A017, and the European Union under the Projects EU-METAMORPHOSE, EU-PHOREMOST, EUPHOME, and EU-ECONAM. One of the authors (E.O.) acknowledges partial support from the Turkish Academy of Sciences.

${ }^{1}$ H. Morkoç, Handbook of Nitride Semiconductors and Devices (WileyVCH, Berlin (2008), Vols. I-III.
${ }^{2}$ J. Xie, J. H. Leach, X. Ni, M. Wu, R. Shimada, Ü. Özgür, and H. Morkoç, Appl. Phys. Lett. 91, 262102 (2007).

${ }^{3}$ M. Miyoshi, T. Egawa, H. Ishikawa, K.-I. Asai, T. Shibata, M. Tanaka, and O. Oda, J. Appl. Phys. 98, 063713 (2005).

${ }^{4}$ J. Xie, X. Ni, M. Wu, J. H. Leach, Ü. Özgür, and H. Morkoç, Appl. Phys. Lett. 91, 132116 (2007).

${ }^{5}$ M. Gonschorek, J.-F. Carlin, E. Feltin, M. A. Py, and N. Grandjean, Appl. Phys. Lett. 89, 062106 (2006).

${ }^{6}$ S. B. Lisesivdin, S. Acar, M. Kasap, S. Ozcelik, S. Gokden, and E. Ozbay, Semicond. Sci. Technol. 22, 543 (2007).

${ }^{7}$ I. P. Smorchkova, L. Chen, T. Mates, L. Shen, S. Heikman, B. Moran, S. Keller, S. P. DenBaars, J. S. Speck, and U. K. Mishra, J. Appl. Phys. 90, 5196 (2001).

${ }^{8}$ O. Ambacher, J. Smart, J. R. Shealy, N. G. Weimann, K. Chu, M. Murphy, W. J. Schaff, L. F. Eastman, R. Dimitrov, L. Wittmer, M. Stutzmann, W. Rieger, and J. Hilsenbeck, J. Appl. Phys. 85, 3222 (1999).

${ }^{9}$ Y. Cao and D. Jena, Appl. Phys. Lett. 90, 182112 (2007).

${ }^{10}$ S. B. Lisesivdin, A. Yildiz, and M. Kasap, Opt. Adv. Mater.-Rapid Comm. 1, 467 (2007).

${ }^{11}$ T. Palacios, S. Rajan, A. Chakraborty, S. Heikman, S. Keller, S. P. DenBaars, and U. K. Mishra, IEEE Trans. Electron Devices 52, 2117 (2005).

${ }^{12}$ J. Kuzmík, IEEE Electron Device Lett. 22, 510 (2001).

${ }^{13}$ J. Kuzmík, Semicond. Sci. Technol. 17, 540 (2002).

${ }^{14}$ C. Hums, J. Bläsing, A. Dadgar, A. Diez, T. Hempel, J. Christen, A. Krost, K. Lorenz, and E. Alves, Appl. Phys. Lett. 90, 022105 (2007).

${ }^{15}$ S. Yamaguchi, M. Kosaki, Y. Watanabe, S. Mochizuki, T. Nakamura, Y. Yukawa, S. Nitta, H. Amano, and I. Akasaki, Phys. Status Solidi A 188, 895 (2001).

${ }^{16}$ O. Katz, D. Mistele, B. Meyler, G. Bahir, and J. Salzman, IEEE Trans. Electron Devices 52, 146 (2005).

${ }^{17}$ A. Dadgar, M. Neuburger, F. Schulze, J. Bläsing, A. Krtschil, I. Daumiller, M. Kunze, K.-M. Günther, H. Witte, A. Diez, E. Kohn, and A. Krost, Phys. Status Solidi A 202, 832 (2005).

${ }^{18}$ K. Jeganathan, M. Shimizub, H. Okumurab, Y. Yanoc, and N. Akutsu, J. Cryst. Growth 304, 342 (2007).

${ }^{19}$ R. Butté, J.-F. Carlin, E. Feltin, M. Gonschorek, S. Nicolay, G. Christmann, D. Simeonov, A. Castiglia, J. Dorsaz, H. J. Buehlmann, S. Christopoulos, G. Baldassarri Höger von Högersthal, A. J. D. Grundy, M. Mosca, C. Pinquier, M. A. Py, F. Demangeot, J. Frandon, P. G. Lagoudakis, J. J. Baumberg, and N. Grandjean, J. Phys. D 40, 6328 (2007). ${ }^{20}$ H. Yu, D. Caliskan, and E. Ozbay, J. Appl. Phys. 100, 033501 (2006).

${ }^{21}$ E. Arslan, M. K. Ozturk, A. Teke, S. Ozcelik, and E. Ozbay, J. Phys. D 41, 155317 (2008)

${ }^{22}$ P. M. Asbeck, E. T. Yu, S. S. Lau, G. J. Sullivan, J. van Hove, and J. Redwing, Electron. Lett. 33, 1230 (1997).

${ }^{23}$ F. Bernardini, V. Fiorentini, and D. Vanderbilt, Phys. Rev. B 56, R10024 (1997); A. F. Wright, J. Appl. Phys. 82, 2833 (1997).

${ }^{24}$ S. Gokden, R. Baran, N. Balkan, and S. Mazzucato, Physica E (Amsterdam) 24, 249 (2004); S. Gokden, A. Ilgaz, N. Balkan, and S. Mazzucato, ibid. 25, 86 (2004) 